\title{
QUADRATURE OVER CURVED SURFACES BY EXTRAPOLATION
}

\author{
J. N. LYNESS
}

\begin{abstract}
In this paper we describe and justify a method for integrating over curved surfaces. This method does not require that the Jacobian be known explicitly. This is a natural extension of extrapolation (or Romberg integration) for planar squares or triangles.
\end{abstract}

\section{INTRODUCTION}

We treat the numerical integration over a curvilinear quadrilateral, $\sigma$, and over a curvilinear triangle, $\tau$. These are embedded in a curved surface, which may be parameterized in the form

$$
\begin{aligned}
& \mathbf{x}=(x, y, z)=\boldsymbol{\Phi}(\mathbf{u})=\boldsymbol{\Phi}(u, v), \\
& x=\phi(u, v) ; y=\psi(u, v) ; z=\chi(u, v) .
\end{aligned}
$$

The region $\sigma$ (or $\tau$ ) is that part of the curved surface that is mapped from the unit square $S$ (or from the unit triangle $T$ ). That is, $\sigma: \Phi(\mathbf{u} \in S$ ) and $\tau: \Phi(\mathbf{u} \in T)$, where $S$ and $T$ are, respectively,

$$
S: \mathbf{u} \in[0,1)^{2}, \quad T: u+v<1, \quad u \geq 0, v \geq 0 .
$$

The method is designed for a situation in which a triangularization (see below) based on the mapping $\Phi$ is readily available; the functions $\phi, \psi$, and $\chi$ and the Jacobian $J$ (see below) need not be known explicitly. However, all these functions, known or unknown, are well behaved, and the surface in which $\sigma$ and $\tau$ are embedded is smooth.

A situation in which this may occur is one in which the surface is defined implicitly:

$$
H(x, y, z)=0,
$$

and $\nabla H$ is available. In some such cases, one may obtain accurate values of $\mathbf{x}$ iteratively, without having to know $\boldsymbol{\Phi}$ explicitly. Georg [3] discusses problems of this sort in the context of the boundary element method. He conjectures the existence of an asymptotic expansion, on which a convenient extrapolation

Received by the editor June 2, 1993 and, in revised form, December 3, 1993.

1991 Mathematics Subject Classification. Primary 65D32, 65B05, 65B15.

Key words and phrases. Cubature, curvilinear triangle, Euler-Maclaurin expansion, extrapolation, Romberg integration.

This work was supported by the Office of Scientific Computing, U.S. Department of Energy, under Contract W-31-109-Eng-38. 
scheme may be based. In this paper, we have been able to establish this expansion. This puts on a sound theoretical footing some of his innovative but partly heuristic work in this area.

We now describe a simple conventional method based on triangularization. Throughout this paper, $m$ is a positive integer. The $m$-triangularization of the planar square $[0,1]^{2}$ comprises a partition into $2 m^{2}$ distinct triangles, using the lines

$$
u=j / m, \quad v=j / m, \quad u+v=j / m \quad \text { for all } j .
$$

The vertices of these triangles comprise a set of $(m+1)^{2}$ points $\left(t_{j}, t_{k}\right)$, where $j, k \in[0, m]$ and

$$
t_{j}=t_{j}(m)=j / m \text {. }
$$

We shall suppress the dependence on $m$ in cases where no confusion is likely to arise.

The mapping function $\mathbf{x}=\boldsymbol{\Phi}(\mathbf{u})$ induces an $m$-triangularization of the curvilinear quadrilateral $\sigma$. The lines (1.3) are mapped into curves embedded in the curved surface, forming a set of $2 \mathrm{~m}^{2}$ curvilinear triangles. The integral $I_{\sigma} f$ may be approximated using as abscissas the $(m+1)^{2}$ distinct points that are vertices of these triangles. Let $\tau_{\mu}, \mu=1,2, \ldots, 2 m^{2}$, represent these curvilinear triangles; let $f_{\mu, 1}, f_{\mu, 2}, f_{\mu, 3}$ represent the function values of $f$ at the three vertices of $\tau_{\mu}$; and let $\bar{\Delta}_{\mu}$ be the area of the planar triangle having these vertices. Then it is evident that

$$
Q^{(m)} f=\frac{1}{3} \sum_{\mu=1}^{2 m^{2}} \bar{\Delta}_{\mu}\left(f_{\mu, 1}+f_{\mu, 2}+f_{\mu, 3}\right)
$$

is an approximation to $I_{\sigma} f$.

The principal result in this paper is that $Q^{(m)} f$ has an $h^{2}$-error expansion. That is, setting $h=1 / m$, when $f \in C^{(2 p)}(\sigma)$ and $\Phi(\mathbf{u})$ is sufficiently well behaved, we have

$$
Q^{(m)} f=I_{\sigma} f+B_{2} h^{2}+B_{4} h^{4}+\cdots+B_{2 p} h^{2 p}+O\left(h^{2 p+1}\right),
$$

where the coefficients $B_{2 q}$ are independent of $h$. In the course of obtaining this result, we uncover several other results of the same nature. Finally, we obtain the result, given in Theorem 5.6 below, which is analogous to (1.6) when the region $\sigma$ is replaced by the curvilinear triangle $\tau$.

This theorem is in fact Conjecture 5.1 of Georg [3], which is itself part of Conjecture 1 of Georg and Tausch [4]. This expansion may be used as a basis for $h^{2}$-extrapolation; see, for example, Bauer, Rutishauser, and Stiefel [1]. One evaluates successively $Q^{(m)} f$ for a sequence of values of $m$ and, either by means of a Neville-Romberg T-table or by some other simple technique, one obtains a sophisticated result based on this sequence of possibly individually inadequate results. Some advantages and other features of this approach form part of the paper by Georg. The present paper is confined to one significant task: to establish the asymptotic expansion on which the underlying theory of his work may be based.

The author recently became aware that this same conjecture was established by Verlinden and Cools [9] simultaneously and independently. Their paper appears in this issue of this journal. 
As a preliminary, in $\S 2$, we describe a method based on extrapolation that one might use if the Jacobian function $J$ in (2.2) below were readily available. The subsequent three sections are devoted to modifying this method so as to avoid the explicit evaluation of $J$. Section 3 is devoted to the surface, delineating the relation between $\bar{\Delta}_{\mu}$ and $J$ in some detail. Section 4 provides results for the curvilinear quadrilateral, and $\S 5$ widens these results to the curvilinear triangle. Details of some Euler-Maclaurin-type expansions for the triangle are provided in an Appendix.

\section{BACKGROUND THEORY}

In a recent article, Schwab and Wendland [7] provide a broad survey and analysis of integration over curved surfaces required in the boundary element method. For the benefit of a wider audience, in this paper we start directly from the classical theory. The general theory of analytic integration over surfaces is treated in Courant [2, pp. 273 et seq.]. We follow, as far as possible, the framework introduced there. We set

$$
\iint_{\sigma} f(x, y, z) d S=\iint_{S} f(\phi(u, v), \psi(u, v), \chi(u, v)) J(u, v) d u d v
$$

where

$$
J^{2}=\left(\phi_{u} \psi_{v}-\psi_{u} \phi_{v}\right)^{2}+\left(\psi_{u} \chi_{v}-\chi_{u} \psi_{v}\right)^{2}+\left(\chi_{u} \phi_{v}-\phi_{u} \chi_{v}\right)^{2} .
$$

In this case, the problem reduces to that of evaluating

$$
I g=\int_{0}^{1} \int_{0}^{1} g(u, v) d u d v
$$

where

$$
g(u, v)=f(\phi(u, v), \psi(u, v), \chi(u, v)) J(u, v) .
$$

There are many ways of integrating over a square. The classical approach (see, for example, Stroud [8]) is by means of Gaussian formulas. A marginally less efficient but occasionally more convenient approach is by extrapolation (also known as Romberg integration), one version of which we now describe. Let

$$
R g=\sum_{i=1}^{\nu} w_{i} g\left(u_{i}, v_{i}\right)
$$

be any quadrature rule for $S$ that integrates a constant function correctly. We define the $m^{2}$-copy of this rule $R$ as

$$
R^{(m)} g=\sum_{i=1}^{\nu} \sum_{j=0}^{m-1} \sum_{k=0}^{m-1} \frac{w_{i}}{m^{2}} g\left(\frac{u_{i}+j}{m}, \frac{v_{i}+k}{m}\right) .
$$

When $g \in C^{p}(S)$, the following minor generalization of the Euler-Maclaurin summation formula is valid:

$$
R^{(m)} g-I g=\sum_{s=1}^{p-1} \frac{B_{s}(R ; g)}{m^{s}}+O\left(m^{-p}\right) .
$$

Note that the coefficients $B_{s}$ are independent of $m$, the mesh ratio. Note also that, in general, this is a full expansion containing all inverse integer powers 
of $m$. However, when the rule $R$ is symmetric about the point $(1 / 2,1 / 2)$, all terms $B_{s}$ with odd $s$ vanish. Further, when $R$ is of polynomial degree $d$, we have also that $B_{s}=0$ for all $s \in[1, d]$. Thus, in a context in which $J(u, v)$ is readily available, an attractive way to carry out integration over a curvilinear quadrilateral is by transformation to a plane square and the use of extrapolation. A conventional rule to use in this context might be the product m-panel trapezoidal rule

$$
R^{(m)} g=\frac{1}{m^{2}} \sum_{j=0}^{m} \sum_{k=0}^{m} g\left(t_{j}, t_{k}\right) .
$$

Here, we can identify the positive integer $m$ as the reciprocal of a step length $h$ and define

$$
t_{j}=t_{j}(m)=j h=j / m .
$$

This is, of course, the $m^{2}$-copy of a four-point rule, which applies equal weight to each of the four vertices. This rule has an even error expansion and is used on occasion in the context of two-dimensional Romberg integration.

The rules with which we shall be concerned are more primitive than this. One of these is a rectangle rule defined by

$$
R_{\lambda} g=g(0,0) \text {. }
$$

Neither this nor its $m$-copy (given in (3.11) below) is symmetric, and the expansion (2.6) above is full. Such a rule would be rarely used in practice. However, it turns out to be convenient to develop the theory in terms of such primitive rules. We shall return to this rule and several variants in $\S 3$.

In the rest of this paper we deal with a context in which $J(u, v)$ is not readily available. We describe how the simple approach given above can be modified to deal with this less transparent situation.

\section{THE $m$-TRIANGULARIZATION}

In this section we are concerned with the relation between individual points of the $m$-triangularization of $\sigma$ and the points that are the vertices of these triangles.

Definition 3.1. Let $\lambda$ be a four-integer index $(\alpha, \beta, \gamma, \delta)$. Let $t_{i}=i / m$ and $\mathbf{x}_{j, k}=\boldsymbol{\Phi}\left(t_{j}, t_{k}\right)$. Then, depending on context, $\Delta_{j, k, \lambda}^{(m)}$ either denotes the triangle having vertices $\left(t_{j}, t_{k}\right),\left(t_{j+\alpha}, t_{k+\beta}\right),\left(t_{j+\gamma}, t_{k+\delta}\right)$, or denotes the area of this triangle; and $\bar{\Delta}_{j, k, \lambda}^{(m)}$ either denotes the planar triangle having vertices $\mathbf{x}_{j, k}, \mathbf{x}_{j+\alpha, k+\beta}, \mathbf{x}_{j+\gamma, k+\delta}$, or denotes the area of that triangle.

Definition 3.2. In the above context,

$$
\operatorname{det} \lambda=\alpha \delta-\beta \gamma, \quad-\lambda=(-\alpha,-\beta,-\gamma,-\delta) \text {. }
$$

When $\operatorname{det} \lambda=0$, the triangle $\Delta_{j, k, \lambda}^{(m)}$ has zero area. Moreover, $(\alpha, \beta, \gamma, \delta)$ and $(\gamma, \delta, \alpha, \beta)$ refer to identical triangles. So, without any compromise, we may consider only indices $\lambda$ for which $\operatorname{det} \lambda$ is a positive integer.

In this paper we shall use only six distinct indices $\lambda$, though much of the theory would allow a general assignment. These six refer to the distinct triangles 
of the triangularization that have $\mathbf{x}_{j, k}$ as a vertex. These indices are those that appear in Definition 3.6 below.

In this section we shall prove various results that are valid for all $\lambda$. We shall confine the details of the proofs to the case $\lambda=(1,0,0,1)$, so that $\bar{\Delta}_{j, k, \lambda}^{(m)}$ is the area of a planar triangle having vertices at $\mathbf{x}_{j, k}, \mathbf{x}_{j+1, k}, \mathbf{x}_{j, k+1}$ (the northeast elementary triangle).

Theorem 3.3. Let $\boldsymbol{\Phi}(\mathbf{u})$ (defined in (1.1)) be $C^{(p)}(S)$, and $\operatorname{det} \lambda=1$. Then

$$
2 \bar{\Delta}_{j, k, \lambda}^{(m)}=h^{2}\left(\delta_{0}+h \delta_{1}+\cdots+h^{p-1} \delta_{p-1}\right)+O\left(h^{p+2}\right),
$$

where $\delta_{0}=J\left(t_{j}, t_{k}\right)$ and $\delta_{s}=\delta_{s, \lambda}\left(t_{j}, t_{k}\right)$; here $\delta_{s, \lambda}(u, v)$ is a function of $u$ and $v$ having continuous partial derivatives of order $p-1-s$.

Proof. We treat only the case $\lambda=(1,0,0,1)$, and within this proof we abbreviate $\bar{\Delta}_{j, k, \lambda}^{(m)}$ to $\bar{\Delta}$. The area of this triangle is one half of the absolute value of the triple vector product

$$
\left|\begin{array}{ccc}
\mathbf{e}_{1} & \mathbf{e}_{2} & \mathbf{e}_{3} \\
\phi\left(t_{j+1}, t_{k}\right)-\phi\left(t_{j}, t_{k}\right) & \psi\left(t_{j+1}, t_{k}\right)-\psi\left(t_{j}, t_{k}\right) & \chi\left(t_{j+1}, t_{k}\right)-\chi\left(t_{j}, t_{k}\right) \\
\phi\left(t_{j}, t_{k+1}\right)-\phi\left(t_{j}, t_{k}\right) & \psi\left(t_{j}, t_{k+1}\right)-\psi\left(t_{j}, t_{k}\right) & \chi\left(t_{j}, t_{k+1}\right)-\chi\left(t_{j}, t_{k}\right)
\end{array}\right| .
$$

We may expand this in the form

$$
\mathbf{e}_{1} D^{(1)}+\mathbf{e}_{2} D^{(2)}+\mathbf{e}_{3} D^{(3)}
$$

giving

$$
4 \bar{\Delta}^{2}=D^{(1) 2}+D^{(2) 2}+D^{(3) 2} .
$$

Since $\phi(u, v)$ and the other components of $P$ are well behaved, we may use the expansion

$$
\phi\left(t_{j+1}, t_{k}\right)-\phi\left(t_{j}, t_{k}\right)=h \phi_{u}+\frac{h^{2}}{2} \phi_{u u}+\cdots
$$

and similar expansions for the other elements of $D^{(3)}$. This gives

where

$$
\begin{aligned}
D^{(3)} & =h^{2}\left|\begin{array}{ll}
\phi_{u}+\frac{h}{2} \phi_{u u}+\cdots & \psi_{u}+\frac{h}{2} \psi_{u u}+\cdots \\
\phi_{v}+\frac{h}{2} \phi_{v v}+\cdots & \psi_{v}+\frac{h}{2} \phi_{v v}+\cdots
\end{array}\right| \\
& =h^{2}\left(D_{0}^{(3)}+h D_{1}^{(3)}+\cdots+h^{p-1} D_{p-1}^{(3)}\right)+O\left(h^{p+2}\right),
\end{aligned}
$$

$$
D_{0}^{(3)}=\left|\begin{array}{c}
\phi_{u} \psi_{u} \\
\phi_{v} \psi_{v}
\end{array}\right|=\frac{\partial(\phi, \psi)}{\partial(u, v)}
$$

and $D_{s}^{(3)}$, the coefficient of $h^{s+2}$ in this expansion, is a sum of analogously constructed products of partial derivatives. It follows that $4 \bar{\Delta}^{2}$ has an expansion in powers of $h$ of the form

$$
4 \bar{\Delta}^{2}=h^{4}\left(J^{2}+\sum_{s=1}^{p-1} h^{s} C_{s}\right)+O\left(h^{p+4}\right) .
$$

Finally, for sufficiently small $h$, we may take the square root of this to obtain

$$
2 \bar{\Delta}=h^{2} J+\sum_{s=1}^{p-1} \delta_{s} h^{s}+O\left(h^{p+2}\right) .
$$


The series manipulation is elementary; to establish the theorem, we need information about the continuity of the functions $\delta_{s, \lambda}(u, v)$.

Since the derivatives of $\phi, \psi$, and $\chi$ of order $p$ are continuous functions of $u$ and $v$, it follows that so are the derivatives of $D_{s}^{(3)}$ of order $p-1-s$; similarly, those of $D_{s}^{(1)}$ and $D_{s}^{(2)}$ have the same order of continuity. Then $C_{s}$ has this order. Finally, so long as $J(u, v)$ is bounded away from zero in the region $S$, we find that $\delta_{s}$ also has this order of continuity.

It is relatively straightforward to show that the effect of replacing $\lambda$ by $-\lambda$ in the above proof is the same as that of reversing the sign of $h$; this gives

$$
\delta_{s, \lambda}(u, v)=(-1)^{s} \delta_{s,-\lambda}(u, v) .
$$

It is no surprise that the first term in expansion (3.1) is $h^{2} J$. This is needed for the classical theory to survive. An expansion in powers of $h$ is not unexpected. What is critical is that the coefficients $\delta_{s, \lambda}(u, v)$ are smooth functions of $u$ and $v$. Although they occur in the form $\delta_{s, \lambda}\left(t_{j}, t_{k}\right)$ and $t_{j}, t_{k}$ depend on $m$, the functions $\delta_{s, \lambda}(u, v)$ do not depend on $m$. Like $J=\delta_{0}(u, v)$, they depend on $\phi, \psi$, and $\chi$ only. This circumstance is exploited later.

The condition $\operatorname{det} \lambda=1$ results in $\delta_{0, \lambda}(u, v)=J(u, v)$. If this were relaxed, the same theorem would hold with $\delta_{0, \lambda}(u, v)=J(u, v) \operatorname{det} \lambda$.

We now discuss the connection between the $m$-triangularization and the indices $\lambda$. The following definition is a first step in clarifying a somewhat involved situation.

Definition 3.4. Let $B$ denote the square $S$ or the triangle $T$ defined in (1.2) Then $\delta_{j, k, \lambda}^{(m)}(B)=1$ or 0 depending on whether or not the triangle $\Delta_{j, k, \lambda}^{(m)}$ forms part of the $m$-triangularization (1.3) of $B$.

By inspection, one can verify that there are only six distinct values of index $\lambda$ for which it is possible for any such triangle to be part of the triangularization. We refer to these six (which are precisely those that appear in Definition 3.6 below) as the triangularization-compatible $(T C)$ indices.

We now return to the construction of quadrature rules.

Definition 3.5. Let $B$ denote $S$ or $T$. We denote by $R_{\lambda}^{(m)}(B)$ the rule that assigns a weight $\mathrm{m}^{-2}$ to all points $\left(t_{j}, t_{k}\right)$ of the $m$-triangularization of $B$ for which the triangle $\Delta_{j, k, \lambda}^{(m)}$ lies in $B$ and is a member of the triangularization.

Definitions 3.4 and 3.5 will be applied in $\S 5$ in a context where $B$ is the triangle $T$. In this section and in $\S 4$ we shall suppress the argument $(S)$. We find that, for the six TC indices, the definition is satisfied as follows.

Definition 3.6. $R_{\lambda}^{(m)}$ is the $m^{2}$-copy of $R_{\lambda}$ given by

$$
\begin{aligned}
R_{(1,0,0,1)} g & =g(0,0), \\
R_{(0,1,-1,1)} & =g(1,0), \\
R_{(-1,1,-1,0)} & =g(1,0), \\
R_{(-1,0,0,-1)} g & =g(1,1), \\
R_{(0,-1,1,-1)} & g=g(0,1), \\
R_{(1,-1,1,0)} & =g(0,1) .
\end{aligned}
$$


Clearly, for no index $\lambda$ is $R_{\lambda}^{(m)}$ the product trapezoidal rule (which is symmetric). It is the $m^{2}$-copy of a one-point rule that assigns full weight to one corner of the unit square. Specifically, in accordance with Definition 3.4,

$$
R_{\lambda}^{(m)} g=\frac{1}{m^{2}} \sum_{j=0}^{m} \sum_{k=0}^{m} \delta_{j, k, \lambda}^{(m)} g\left(t_{j}, t_{k}\right),
$$

where $\delta_{j, k, \lambda}^{(m)}=1$ for only $m^{2}$ of the $(m+1)^{2}$ specified abscissas in (3.11), and is zero otherwise. For example,

$$
R_{(1,0,0,1)}^{(m)} g=\frac{1}{m^{2}} \sum_{j=0}^{m-1} \sum_{k=0}^{m-1} g\left(t_{j}, t_{k}\right) .
$$

However, since it is a copy rule, the full Euler-Maclaurin expansion (2.6) applies to this rule.

Corresponding to each $R_{\lambda}^{(m)}$ we now define a quadrature rule for the curvilinear quadrilateral $\sigma$. This is obtained by first transforming the rule onto the curved surface and then replacing the Jacobian-dependent term by the area of an elementary triangle. The transformation gives

$$
R_{\lambda}^{(m)} g=\sum_{j=0}^{m} \sum_{k=0}^{m} \delta_{j, k, \lambda}^{(m)} h^{2} J\left(t_{j}, t_{k}\right) f\left(\mathbf{x}_{j, k}\right),
$$

and the arbitrary replacement leads to the following definition.

Definition 3.7. We let

$$
Q_{\lambda}^{(m)} f=\sum_{j=0}^{m} \sum_{k=0}^{m} \delta_{j, k, \lambda}^{(m)} 2 \bar{\Delta}_{j, k, \lambda}^{(m)} f\left(\mathbf{x}_{j, k}\right) .
$$

Although this appears to contain $(m+1)^{2}$ abscissas, in fact only $m^{2}$ have nonzero weights. For example, in the case $\lambda=(1,0,0,1)$, abscissas with $j=m$ and with $k=m$ have zero weights.

Note carefully that, in spite of the notation, the rule in (3.14) is not an $\mathrm{m}^{2}$ copy rule. It is obtained by modifying a term in a rule that is an $\mathrm{m}^{2}$-copy rule in a different space. The expansion of Theorem 3.3 allows us to establish the following.

Theorem 3.8. Let $\Phi(\mathbf{u})$ (defined in (1.1)) be $C^{(p)}(S)$, and let $Q_{\lambda}^{(m)} f$ and $R_{\lambda}^{(m)} g$ be given by (3.14) and (3.11), respectively. Then

$$
Q_{\lambda}^{(m)} f=\sum_{s=0}^{p-1} h^{s} R_{\lambda}^{(m)} g_{s, \lambda}+O\left(h^{p}\right)
$$

where

$$
g_{s, \lambda}(u, v)=f(\mathbf{x}(u, v)) \delta_{s, \lambda}(u, v)
$$

and the functions $\delta_{s, \lambda}$ are defined in Theorem 3.3.

Proof. This is simply a matter of subsituting for $\bar{\Delta}_{j, k, \lambda}^{(m)}$ in (3.14) the expression given in (3.1) and simplifying by using (3.11). 
Note that $g_{0, \lambda}(u, v)$ coincides with $g(u, v)$. Note also that (3.15) is not an $h$-expansion, since $R_{\lambda}^{(m)} g_{s, \lambda}$ depends on $h$ through $m$.

We have, as yet, made no commitment about the properties of $f$ or $g$. To proceed, we need to be specific about the form of $g_{s, \lambda}(u, v)$. In the rest of this paper we treat the case in which $f(\mathbf{x})$, and consequently $g_{s, \lambda}(u, v)$, are well behaved.

\section{RULES FOR THE CURVILINEAR QUADRILATERAL}

Theorem 3.8 expresses $Q_{\lambda}^{(m)} f$, a rule over the curved surface $\sigma$, in an expansion, each term of which involves a product rectangle rule evaluation over the planar square $S$. As mentioned above, this is not an $h$-expansion.

We now restrict ourselves to an integrand $g \in C^{(p)}(S)$. In this case we may make use of the Euler-Maclaurin expansion (2.6) applied to each rule $R_{\lambda}$ in this expansion. This expansion is of the form

$$
R_{\lambda}^{(m)} g=\sum_{j=0}^{p-1} h^{j} B_{j}\left(R_{\lambda} ; g\right)+O\left(m^{-p}\right) .
$$

Here, we have set $h=1 / m$; bearing in mind that

$$
B_{0}(R ; g)=I(g) \equiv \int_{0}^{1} \int_{0}^{1} g(u, v) d u d v
$$

and that $B_{j}(R ; g)$ is independent of $m$, we may substitute (4.1) into (3.15) to establish the following theorem.

Theorem 4.1. When $\boldsymbol{\Phi}(\mathbf{u})$ (defined in (1.1)) and $f\left(\mathbf{x}(u, v)\right.$ ) are $C^{(p)}(S)$, the quadrature rule $Q_{\lambda}^{(m)} f$ of Definition 3.7 has an h-error expansion, namely,

$$
Q_{\lambda}^{(m)} f-I_{\sigma} f=\sum_{w=1}^{p-1} \frac{\widetilde{B}_{w}(\lambda ; f)}{m^{w}}+O\left(h^{p}\right),
$$

where

$$
\widetilde{B}_{w}(\lambda ; f)=\sum_{j=0}^{w} B_{j}\left(R_{\lambda} ; g_{w-j, \lambda}\right) .
$$

When $\lambda$ is one of the six TC indices, the rule described by this theorem has the property that the points for function evaluation lie in the completion of $\sigma$.

Any one of these rules alone seems quite reasonable, but a little "lop-sided". In the context of extrapolation, a rule having an $h^{2}$-error expansion would be preferable.

The rest of this section is devoted to deriving such a rule. It turns out (see Theorem 4.4 below) that the average of the six rules mentioned above has an $h^{2}$-error expansion.

Lemma 4.2. The coefficients in the Euler-Maclaurin expansion (4.1) satisfy

$$
B_{j}\left(R_{\lambda} ; g\right)=(-1)^{j} B_{j}\left(R_{-\lambda} ; g\right) \text {. }
$$

Proof. The result may be established by direct evaluation of the coefficients in terms of Bernoulli functions. A more elegant approach is based on the circumstance that a symmetric rule has an $\mathrm{m}^{2}$-error expansion (and an antisymmetric 
rule operator has an expansion involving only odd powers of $m$ ). It is readily verified that

$$
R g=\frac{1}{2} R_{\lambda} g+\frac{1}{2} R_{-\lambda} g
$$

is symmetric. When $j$ is odd, $B_{j}(R ; g)$ vanishes, leaving

$$
B_{j}\left(R_{-\lambda}, g\right)=-B_{j}\left(R_{\lambda}, g\right) \text { for all odd } j .
$$

A corresponding argument provides a corresponding result for even $j$, thus establishing (4.5).

Another simple proof using an integral representation of $B_{j}\left(R_{\lambda} ; g\right)$ is outlined in the appendix.

A feature of the foregoing theory, which has been specially built in, is that in many $h$-expansions involving $\lambda$, the effect of reversing the sign of $h$ is the same as that of reversing the sign of $\lambda$. In particular, in view of (3.4), the functions defined in (3.16) satisfy

$$
g_{s, \lambda}(u, v)=(-1)^{s} g_{s,-\lambda}(u, v) .
$$

We are now ready to consider the rule defined in (4.8) below. This rule applies to each fully interior point $\mathbf{x}_{i, j}$ a weight equal to the sum of the areas of two opposite triangles. (For example, when $\lambda=(1,0,0,1)$, these are the one to the northeast and the one to the southwest.) In general, points on edges have weights involving only one of these triangles, the interior one. Two of the vertices have zero weight.

Theorem 4.3. Under the hypotheses of Theorem 4.1 the quadrature rule

$$
Q^{(m)} f=\frac{1}{2} Q_{\lambda}^{(m)} f+\frac{1}{2} Q_{-\lambda}^{(m)} f
$$

has an $h^{2}$-error expansion.

Proof. The proof relies entirely on equations (4.5) and (4.7). These are used in (4.4) to show

$$
\begin{aligned}
\widetilde{B}_{w}(\lambda ; f) & =\sum_{j=0}^{w} B_{j}\left(R_{\lambda} ; g_{w-j, \lambda}\right) \\
& =\sum_{j=0}^{w}(-1)^{j}(-1)^{w-j} B_{j}\left(R_{-\lambda} ; g_{w-j,-\lambda}\right)=(-1)^{w} \widetilde{B}_{w}(-\lambda ; f) .
\end{aligned}
$$

The error expansion for $Q^{(m)} f$ in (4.8) is the average of two asymptotic series of the form (4.3), the second differing from the first only in that $\lambda$ is replaced by $-\lambda$. Thus, the coefficient of $m^{-w}$ in this expansion is $\frac{1}{2}\left(\widetilde{B}_{w}(\lambda ; f)+\widetilde{B}_{w}(-\lambda ; f)\right)$. In view of $(4.9)$, this coefficient vanishes when $w$ is odd. This establishes the theorem.

Our final result for the curvilinear quadrilateral is as follows.

Theorem 4.4. Under the hypotheses of Theorem 4.1 the quadrature rule

$$
\begin{aligned}
& Q^{(m)} f=\frac{1}{6}\left(Q_{(1,0,0,1)}^{(m)} f+Q_{(0,1,-1,1)}^{(m)} f+Q_{(-1,1,-1,0)}^{(m)} f\right. \\
& \left.\quad+Q_{(-1,0,0,-1)}^{(m)} f+Q_{(0,-1,1,-1)}^{(m)} f+Q_{(1,-1,1,0)}^{(m)} f\right)
\end{aligned}
$$

has an $h^{2}$-error expansion. 
Proof. The proof is a simple corollary of Theorem 4.3. The quadrature rule here is obtained by taking the mean of three rules, each being of form (4.8); specifically, the three values of $\lambda$ involved are those in the first three assignments in (4.10). Naturally, the mean of three $h^{2}$-error expansions is itself an $h^{2}$-error expansion.

This rule applies to each point $\mathbf{x}_{j, k}$ a contribution from each of the triangles inside $\sigma$ of which it is a vertex. This contribution is one-third of the planar area of the triangle in question. Thus, it could be reexpressed in terms of contributions from triangles. Doing this, reveals the expression in (1.5). Thus, this theorem is one of the principal results of this paper, foreshadowed in the introduction.

\section{RULES FOR THE CURVILINEAR TRIANGLE}

In this section we treat the numerical integration over the curvilinear triangle $\tau$, which, we recall from $\S 1$, is embedded in the same curved surface (1.1) as is $\sigma$. Thus, much of the theory in $\S \S 1-3$ applies with only minor modification. The region $\tau$ is that part of the curved surface that is mapped from the unit triangle. That is, $\tau: \Phi(\mathbf{u} \in T)$, where $T$ is

$$
T: u+v<1, \quad u \geq 0, v \geq 0 \text {. }
$$

The same triangularization (1.3) is used, and so is the same notation introduced in $\S 3$ to describe individual triangles: $m^{2}$ of the triangles in the $m$ triangularization of $S$ form the $m$-triangularization of $T$.

We may construct a rule for $T$ using the technique of $\S 3$. We use Definition 3.4 for $\delta_{j, k \lambda}^{(m)}(T)$ and then define $R_{\lambda}^{(m)}(T)$ in accordance with Definition 3.5. This gives

$$
R_{\lambda}^{(m)}(T) g=\frac{1}{m^{2}} \sum_{j=0}^{m} \sum_{k=0}^{m} \delta_{j, k, \lambda}^{(m)}(T) g\left(t_{j}, t_{k}\right) .
$$

Following the same steps as in $\S 3$, we are led to the correspondent of Definition 3.7.

Definition 5.1. We let

$$
Q_{\lambda}^{(m)}(\tau) f=\sum_{j=0}^{m} \sum_{k=0}^{m} \delta_{j, k, \lambda}^{(m)}(T) 2 \bar{\Delta}_{j, k, \lambda}^{(m)} f\left(\mathbf{x}_{j, k}\right) .
$$

One may verify that, when $\lambda$ is one of the six TC indices, this employs either $m(m+1) / 2$ or $m(m-1) / 2$ function values. The result of Theorem 3.8 in this context is simply

$$
Q_{\lambda}^{(m)}(\tau) f=\sum_{s=0}^{p-1} h^{s} R_{\lambda}^{(m)}(T) g_{s, \lambda}+O\left(h^{p}\right)
$$

where, as before,

$$
g_{s, \lambda}(u, v)=f(\mathbf{x}(u, v)) \delta_{s, \lambda}(u, v)
$$

and the functions $\delta_{s, \lambda}$ are defined in Theorem 3.3. 
In $\S 4$ we proceeded by invoking the two-dimensional Euler-Maclaurin expansion for the square. Less well known is the corresponding formula for the triangle. Besides the overall formula, we shall also require some details about the structure of the coefficients. The following theorem is a specialization of one given in Lyness and Puri [6].

Theorem 5.2. When $g \in C^{(p)}(T)$, then

$$
R_{\lambda}^{(m)}(T) g=\sum_{j=0}^{p-1} h^{j} B_{j}\left(R_{\lambda} ; T ; g\right)+O\left(m^{-p}\right)
$$

where

$$
B_{0}\left(R_{\lambda} ; T ; g\right)=I(T ; g) \equiv \iint_{T} g(u, v) d u d v .
$$

An integral representation for $B_{j}\left(R_{\lambda} ; T ; g\right)$ is given in the appendix. We now substitute (5.6) into (5.4) to obtain the following theorem.

Theorem 5.3. Under the hypotheses of Theorem 4.1 the quadrature rule $Q_{\lambda}^{(m)}(\tau) f$ of Definition 5.1 has an h-error expansion, namely,

$$
Q_{\lambda}^{(m)}(\tau) f-I_{\tau} f=\sum_{w=1}^{p-1} \frac{\widetilde{B}_{w}(\lambda ; \tau ; f)}{m^{w}}+O\left(h^{p}\right),
$$

where

$$
\widetilde{B}_{w}(\lambda ; \tau ; f)=\sum_{j=0}^{w} B_{j}\left(R_{\lambda} ; T ; g_{w-j, \lambda}\right) .
$$

It remains to establish quadrature rules having $h^{2}$-error expansions. We follow precisely the same approach as in the preceding section for the square. We need the following result, which corresponds to Lemma 4.2 and relates coefficients in different Euler-Maclaurin expansions.

Lemma 5.4. The coefficients in the Euler-Maclaurin expansion (5.6) satisfy

$$
B_{j}\left(R_{\lambda} ; T ; g\right)=(-1)^{j} B_{j}\left(R_{-\lambda} ; T ; g\right) .
$$

This is significantly more difficult to prove than the corresponding result for the square, and we defer this to the appendix. However, once this is established, the rest of the theory follows in a relatively straightforward manner. Corresponding to Theorem 4.3, we have the following theorem.

Theorem 5.5. Under the hypotheses of Theorem 4.1 the quadrature rule

$$
Q^{(m)}(\tau) f=\frac{1}{2} Q_{\lambda}^{(m)}(\tau) f+\frac{1}{2} Q_{-\lambda}^{(m)}(\tau) f
$$

has an $h^{2}$-error expansion.

Proof. Exactly as in the proof of Theorem 4.3 we use (5.9), (5.10), and (4.7) to show that $\frac{1}{2}\left(\widetilde{B}_{w}(\lambda ; \tau ; f)+\widetilde{B}_{w}(-\lambda ; \tau ; f)\right)$ vanishes when $w$ is odd. Since this is the coefficient of $m^{-w}$ in the error expansion of $Q^{(m)}(\tau) f$, the theorem is established.

Our final result for the curvilinear triangle is as follows. 
Theorem 5.6. Under the hypotheses of Theorem 4.1 the quadrature rule (5.12)

$$
\begin{aligned}
Q^{(m)}(\tau) f=\frac{1}{6}\left(Q_{(1,0,0,1)}^{(m)}(\tau) f+Q_{(0,1,-1,1)}^{(m)}(\tau) f+Q_{(-1,1,-1,0)}^{(m)}(\tau) f\right. \\
\left.\quad+Q_{(-1,0,0,-1)}^{(m)}(\tau) f+Q_{(0,-1,1,-1)}^{(m)}(\tau) f+Q_{(1,-1,1,0)}^{(m)}(\tau) f\right)
\end{aligned}
$$

has an $h^{2}$-error expansion.

This rule is simply the mean of three examples of the rule in Theorem 5.5. These three have the indices $\lambda$ coinciding with the first three occurring in (5.12). The mean of three rules each of which has an $h^{2}$-error expansion also has an $h^{2}$-error expansion. As mentioned in $\S 1$, this theorem coincides with Conjecture 5.1 of Georg [3] and with part of Conjecture 1 of Georg and Tausch [4].

\section{APPENDIX: Notes ON EULER-MaClaURIN EXPANSIONS FOR PLANAR REGIONS}

The coefficients in the Euler-Maclaurin expansion (2.6) for the square, $S$, for any rule $(2.5)$ (weights $w_{i}$, abscissas $\left(u_{i}, v_{i}\right)$ ) have the following simple integral representation:

$$
B_{j}(R ; g)=\sum_{\substack{j_{1}+j_{2}=j \\ j_{i} \geq 0}} c_{j_{1}, j_{2}}(R) \int_{S} g^{\left(j_{1}, j_{2}\right)}(u, v) d u d v,
$$

where

$$
c_{j_{1}, j_{2}}(R)=\sum_{i=1}^{\nu} w_{i} \frac{B_{j_{1}}\left(u_{i}\right) B_{j_{2}}\left(v_{i}\right)}{j_{1} ! j_{2} !}
$$

is essentially the result of applying the rule $R$ to the product of two Bernoulli polynomials.

For the rules $R_{\lambda}$, defined in Definition 3.6, the coefficients $B_{j}\left(R_{\lambda} ; g\right)$ in (4.1) are of the same form. Using the properties of the Bernoulli polynomials, we find

$$
c_{j_{1}, j_{2}}\left(R_{\lambda}\right)=\frac{B_{j_{1}}(\zeta) B_{j_{2}}(\eta)}{j_{1} ! j_{2} !},
$$

where $\zeta, \eta$ are individually 0 or 1 and $(\zeta, \eta)$ occurs as an argument of $g$ in equations $(3.5)$ to $(3.10)$.

Lemma 4.2 follows from (A.1) and (A.3) as a consequence of the symmetry properties of the Bernoulli polynomials, namely, $B_{j}(0)=(-1)^{j} B_{j}(1)$.

The Euler-Maclaurin expansion for the triangle, $T$, is less known and is dealt with at length in Lyness and Puri [6] and in Lyness [5]. In this case the coefficients have a more complicated structure than that in (A.1). However, in the special case of these trapezoidal-type rules $R_{\lambda}$, a simpler formula is available. For example, when $\lambda=(1,0,0,1)$,

$$
B_{j}\left(R_{\lambda} ; T ; g\right)=\sum_{\substack{j_{1}+j_{2}=j \\ j_{i} \geq 0}} c_{j_{1}, j_{2}}\left(R_{\lambda}\right) \int_{0}^{1} \frac{\partial^{j_{1}}}{\partial u^{j_{1}}}\left\{\int_{0}^{1-u} \frac{\partial^{j_{2}}}{\partial v^{j_{2}}} g d v\right\} d u
$$

with $c_{j_{1}, j_{2}}\left(R_{\lambda}\right)$ given by (A.3) above. When $j_{1}=0$, the $j_{1}, j_{2}$-term in the sum reduces to an integral over $T$, in direct analogy to (A.1) above. But otherwise 
the effect of differentiation with respect to the limit of an integral is to introduce additional terms.

In $\S 5$ the derivation of curvilinear rules $Q(\tau) f$ having $h^{2}$-error expansions rested on Lemma 5.4. We shall establish this lemma by showing two lemmas.

Lemma A.1. The operator (null rule) defined by

$$
R_{\lambda-}^{(m)}(T) g=\frac{1}{2}\left\{R_{\lambda}^{(m)}(T) g-R_{-\lambda}^{(m)}(T) g\right\}
$$

has an error expansion involving only odd powers of $h=1 / \mathrm{m}$.

Lemma A.2. The rule defined by

$$
R_{\lambda+}^{(m)}(T) g=\frac{1}{2}\left\{R_{\lambda}^{(m)}(T) g+R_{-\lambda}^{(m)}(T) g\right\}
$$

has an $h^{2}$-error expansion.

Proof. We treat only the case $\lambda=(1,0,0,1)$. We recall that both $R_{\lambda}^{(m)}$ and $R_{-\lambda}^{(m)}$ apply a weight $1 / m^{2}$ to every strictly interior point. In addition, $R_{\lambda}^{(m)}$ applies this weight to all points on edges $x=0$ and $y=0$, except for points $(1,0)$ and $(0,1)$. On the other hand, $R_{-\lambda}^{(m)}$ applies the same weight only to all points on the edge $x+y=1$, except for the endpoints. Applying these assignments to the operators in (A.5) and (A.6), we find

$$
\begin{aligned}
R_{\lambda+}^{(m)} g= & \frac{1}{m^{2}} \sum_{\substack{j+k<m \\
j>k>0}} g\left(\frac{j}{m}, \frac{k}{m}\right) \\
& +\frac{1}{2 m^{2}} \sum_{j=1}^{m-1}\left(g\left(\frac{j}{m}, 0\right)+g\left(0, \frac{j}{m}\right)+g\left(\frac{j}{m}, \frac{m-j}{m}\right)\right) \\
& +\frac{1}{2 m^{2}} g(0,0),
\end{aligned}
$$

while

$$
\begin{aligned}
R_{\lambda-}^{(m)} g= & \frac{1}{2 m^{2}} \sum_{j=1}^{m-1}\left(g\left(\frac{j}{m}, 0\right)+g\left(0, \frac{j}{m}\right)-g\left(\frac{j}{m}, \frac{m-j}{m}\right)\right) \\
& +\frac{1}{2 m^{2}} g(0,0),
\end{aligned}
$$

which can be reexpressed in the form

$$
2 m R_{\lambda-}^{(m)} g=\frac{1}{m} \sum_{j=0}^{m} \prime\left(g\left(\frac{j}{m}, 0\right)+g\left(0, \frac{j}{m}\right)-g\left(\frac{j}{m}, \frac{m-j}{m}\right)\right) .
$$

The right-hand side comprises three one-dimensional trapezoidal rule operators; since these are symmetric, each has an $h^{2}$-error expansion. Thus, the expansion for $R_{\lambda-}^{(m)} g$ involves only odd inverse powers of $m$, establishing Lemma A.1.

In Lyness and Puri $[6, \S 7]$, rules for the triangle of the same general nature as (A.7) are discussed in detail. In particular, a rule denoted there by $R_{s}^{(m)} g$ is defined that has an even expansion. This rule differs from $R_{\lambda+}^{(m)}(T) g$ above only in that the weights attached to vertices are different. Specifically,

$$
R_{s}^{(m)} g=R_{\lambda+}^{(m)}(T) g+\frac{1}{8 m^{2}}(g(1,0)+g(0,1)-2 g(0,0)) .
$$


These variant weights simply modify the coefficient of $1 / \mathrm{m}^{2}$ in the expansion; its even nature is, of course, not compromised. This establishes Lemma A.2 above.

As a convenience to the reader, we note that equations (3.5) through (3.10) may be abbreviated to

$$
R_{(\alpha, \beta, \gamma, \delta)} g=g(\zeta, \eta),
$$

where, so long as $\operatorname{det} \lambda=1$, we have

$$
\begin{aligned}
& \zeta=1 \text { or } 0 \text { according as } \beta>\alpha \text { or } \beta<\alpha, \\
& \eta=1 \text { or } 0 \text { according as } \gamma>\delta \text { or } \gamma<\delta .
\end{aligned}
$$

Note that $\beta+\gamma=0, \alpha \neq \beta, \gamma \neq \delta, \zeta-\eta=\alpha+\beta-\gamma-\delta$.

\section{BIBLIOGRAPHY}

1. F. L. Bauer, H. Rutishauser, and E. Stiefel, New aspects in numerical quadrature, Proc. Sympos. Appl. Math., vol. 15, Amer. Math. Soc., Providence, RI, 1963, pp. 199-218.

2. R. Courant, Differential and integral calculus, Vol. 2, Interscience, New York, 1956.

3. K. Georg, Approximation of integrals for boundary element methods, SIAM J. Sci. Statist. Comput. 12 (1991), 443-453.

4. K. Georg and J. Tausch, Some error estimates for the numerical approximation of surface integrals, Math. Comp. 62 (1994), 755-763.

5. J. N. Lyness, Quadrature over a simplex, SIAM J. Numer. Anal. 15 (1978), 122-133, 870-877.

6. J. N. Lyness and K. K. Puri, The Euler-Maclaurin expansion for the simplex, Math. Comp. 27 (1973), 273-293.

7. C. Schwab and W. L. Wendland, On numerical cubatures of singular surface integrals in boundary element methods, Numer. Math. 62 (1992), 343-369.

8. A. H. Stroud, Approximate calculation of multiple integrals, Prentice-Hall, Englewood Cliffs, NJ, 1971.

9. P. Verlinden and R. Cools, Proof of a conjectured asymptotic expansion for the approximation of surface integrals, Math. Comp. 63 (1994), 717-725.

Mathematics and Computer Science Division, Argonne National laboratory, 9700 South Cass Avenue, ARgonne, Illinois 60439

E-mail address: lyness@mcs.anl.gov 